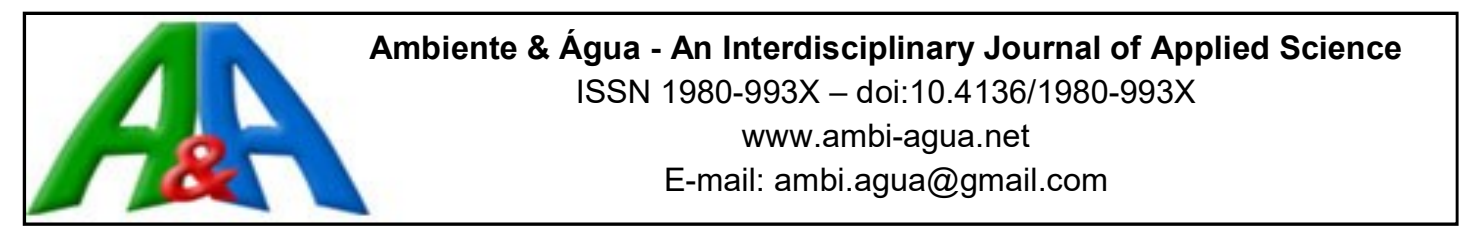

\title{
Teor de nutrientes em folhas de milho fertilizado com vermicomposto de lodo de curtume e irrigado com água residuária doméstica
}

doi:10.4136/ambi-agua.1680

Received: 12 jun. 2015; Accepted: 11 Jul. 2016

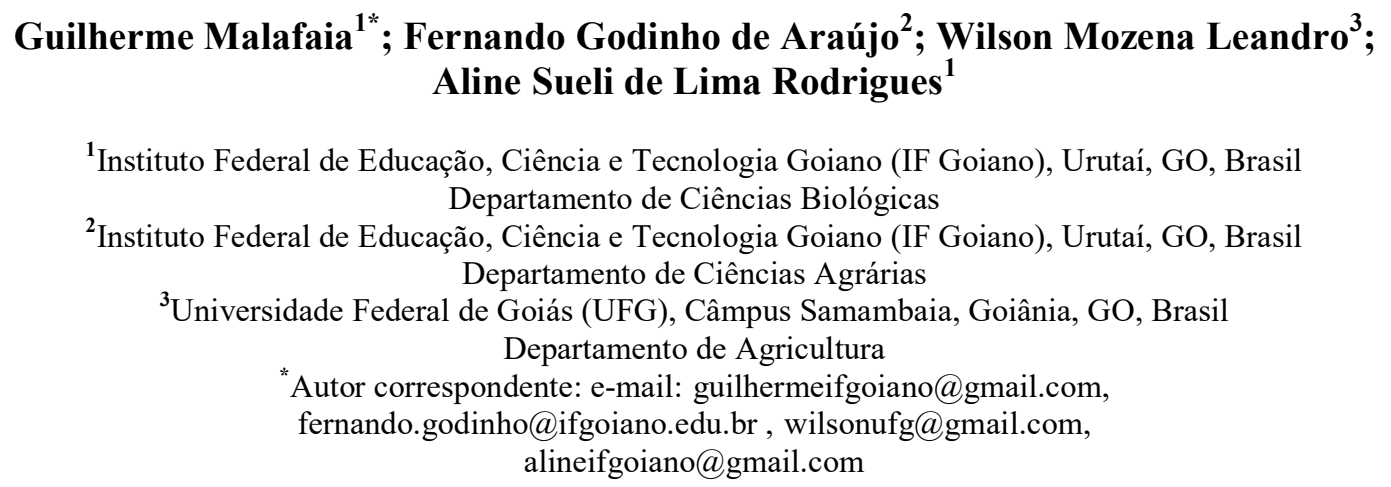

\section{RESUMO}

No presente estudo objetivou-se analisar o teor de macro e micronutrientes de folhas de milho (Zea mays L.) cultivado em solo fertilizado com vermicomposto de lodo de curtume e irrigado com água residuária doméstica. $\mathrm{O}$ arranjo dos tratamentos consistiu em um fatorial 2x6, sendo dois tipos de irrigação: água de abastecimento (A) e água residuária doméstica (R); e seis tipos de adubação: T1: Solo controle, sem adubação química e sem vermicomposto; T2: Solo + NPK; T3: Solo + vermicomposto de lodo primário; T4: Solo + P + vermicomposto de lodo primário; T5: Solo + P + vermicomposto de lodo de caleiro e T6: Solo + vermicomposto de lodo de caleiro, em delineamento inteiramente casualizado (DIC), com cinco repetições. Para a análise do tecido foliar coletou-se, de cada planta, a folha inteira oposta e abaixo da primeira espiga (superior), excluída a nervura central por ocasião do aparecimento da inflorescência feminina. Os resultados apontam que tanto a água residuária quanto o vermicomposto de lodo de curtume constituem boa fonte de nutrientes para as plantas de milho, uma vez que os teores de macro e micronutrientes nas folhas mostraram-se satisfatórios, não tendo sido observado nenhum sinal ou sintoma de toxidez. Apesar de certa fragilidade da análise foliar servir como ferramenta exclusiva para avaliação do estado nutricional das plantas, este estudo, pioneiramente, aponta para a potencialidade do uso da água residuária de origem doméstica e de vermicomposto de lodo de curtume (em associação) na cultura do milho, motivando a realização de novas pesquisas.

Palavras-chave: macronutrientes, micronutrientes, resíduos agroindustriais, reuso de água.

\section{Nutrient content in maize fertilized with tannery sludge vermicompost and irrigated with domestic wastewater}

\section{ABSTRACT}

This study analyzed the macro and micronutrient content of maize leaves (Zea mays L.) 
grown in soil containing tannery sludge vermicomposting and irrigated with wastewater. The arrangement of the treatments consisted of a factorial $2 \times 6$ (two types of irrigation and six kinds of fertilizer) in a completely randomized design, with five repetitions, totaling sixty experimental units. The following experimental units, irrigated with supply water (A) and household wastewater (R), were established: (T1) Control Soil, with no chemical fertilization and no vermicomposting; (T2) Soil + NPK; (T3) Soil + primary sludge vermicompost; (T4) Soil + P + primary sludge vermicompost; (T5) Soil + P + liming sludge vermicompost; and (T6) Soil + liming sludge vermicompost. For the leaf-tissue analysis, the opposite whole leaf below the first (upper) ear was collected from each plant, excluding the midrib at the onset of the female inflorescence. The results showed that both wastewater and the tannery sludge vermicomposts can be a good source of nutrients for maize plants, since the macro and micronutrients in the leaves of plants were satisfactory and no signs or symptoms of toxicity were observed. While leaf analysis alone is insufficient to assess the nutritional status of plants, this study innovatively suggests the potential beneficial use of a combination of wastewater and tannery sludge vermicompost in the cultivation of corn, motivating new research.

Keywords: agro-industrial wastes, macronutrients, micronutrients, wastewater reuse.

\section{INTRODUÇÃO}

Atualmente, tem sido cada vez mais estimulado o desenvolvimento de pesquisas que visam, além do tratamento, o aproveitamento dos resíduos produzidos pelas atividades agroindustriais. As questões ambientais, em especial, têm suscitado reflexões e preocupações, uma vez que os resíduos gerados têm potencial para causar danos ambientais, se não forem devidamente tratados ou destinados (Kraemer, 2014). Nesse sentido, com o intuito de solucionar ou minimizar essa questão, o aproveitamento de resíduos tem emergido como alternativa interessante e ambientalmente sustentável (Nunes et al., 2009).

Uma atividade geradora de resíduo potencialmente tóxico muito comum no Brasil é o processamento do couro bovino realizado pelas indústrias curtumeiras (Gödecke et al., 2012). A atividade curtumeira faz uso de diversos insumos químicos, os quais muitas vezes são potencialmente poluidores (Luersen et al., 2012). Além disso, gera grandes volumes de resíduos orgânicos e ricos em diversos nutrientes e, por esse motivo, esses resíduos têm sido considerados para o uso agronômico (Gödecke et al., 2012). No entanto, a aplicação desses resíduos de forma "in natura" no solo tem causado controvérsias e resultados divergentes em diferentes culturas agrícolas, uma vez que, em função das características químicas destes resíduos, os mesmos podem influenciar negativamente as características do solo (incluindo sua biota) e não apresentar efeitos desejáveis nas culturas. Para tanto, autores como Vig et al. (2011) e, mais recentemente, Malafaia et al. (2015a; 2015b) sugerem que o material seja previamente vermicompostado, a fim de evitar a contaminação do solo, transformando tais resíduos em compostos orgânicos nobres.

Outro resíduo de interesse corresponde às águas residuárias geradas nas atividades domésticas. Com o surgimento de conflitos pelo uso da água e em função da quantidade expressiva demandada pela irrigação, o interesse pelo uso de efluentes tratados em substituição ou complementação às fontes normalmente utilizadas para irrigação tem aumentado. Diferentes estudos têm apontado para o potencial uso dessas águas na agricultura, por estas águas aumentarem, por exemplo, o pH de solos ácidos, concentrações de $\mathrm{P}$ e $\mathrm{S}$ disponível, $\mathrm{K}^{+}, \mathrm{Ca}^{2+}, \mathrm{Mg}^{2+}$ trocáveis, $\mathrm{MO}, \mathrm{N}$-total e diminuição da acidez trocável (Leal et al., 2011; Fonseca et al., 2011; Andrade-Filho et al., 2013; Bonini et al., 2014; Silva et al., 2014; Monteiro et al., 2014). 
Contudo, o uso de resíduos de curtume combinado com a utilização de águas na irrigação das culturas carece de informações sobre a influência que os componentes presentes nestes resíduos podem exercer no desenvolvimento das plantas. A diagnose foliar é uma estratégia interessante para avaliar o estado nutricional das plantas, que por sua vez está diretamente ligado às condições encontradas no solo e à adubação, embora não deva ser considerada ferramenta exclusiva para avaliação do estado nutricional das plantas. Neste sentido, o presente estudo, pioneiramente, objetivou avaliar os teores de nutrientes em folhas de milho (Zea mays L.) fertilizado com vermicomposto de lodo de curtume e irrigado com água residuária doméstica.

\section{MATERIAL E MÉTODOS}

O estudo foi conduzido em estufa localizada no Instituto Federal Goiano (IF Goiano) Campus Urutaí (GO, Brasil). O solo utilizado no experimento foi retirado da camada superficial $(0-20 \mathrm{~cm})$ de uma área (não natural) localizada próxima à estufa, tendo sido classificado por pesquisadores do Laboratório de Solos e Nutrição de Plantas do IF Goiano Campus Urutaí, como Latossolo Vermelho Distrófico Típico, conforme normas da Embrapa (2013). As características do solo utilizado podem ser observadas na Tabela 1.

Tabela 1. Principais características iniciais do solo e dos vermicompostos de lodo de curtume utilizados no presente estudo. Urutaí, GO, 2016.

\begin{tabular}{|c|c|c|c|}
\hline Variáveis & Solo & $\begin{array}{l}\text { Vermicomposto } \\
\text { (Lc) }^{*}\end{array}$ & $\begin{array}{c}\text { Vermicomposto } \\
\text { (Lp) }^{*}\end{array}$ \\
\hline$\overline{\mathrm{pH}}\left(\mathrm{CaCl}_{2}\right)$ & 5,30 & 8,8 & 8,8 \\
\hline $\mathrm{N}\left(\mathrm{g} \mathrm{kg}^{-1}\right)$ & 0,11 & 1,5 & 1,2 \\
\hline $\mathrm{P}\left(\right.$ Mehlich $\left.-\mathrm{mg} \mathrm{dm}^{-3}\right)$ & 5,00 & 700,0 & 400,0 \\
\hline $\mathrm{K}\left(\mathrm{mg} \mathrm{dm}^{-3}\right)$ & 240,00 & $18.000,0$ & $20.000,0$ \\
\hline $\mathrm{Ca}\left(\mathrm{cmol}_{\mathrm{c}} \mathrm{dm}^{-3}\right)$ & 2,60 & 14,0 & 14,0 \\
\hline $\operatorname{Mg}\left(\mathrm{cmol}_{\mathrm{c}} \mathrm{dm}^{-3}\right)$ & 0,80 & 18,0 & 15,0 \\
\hline CTC $\left(\mathrm{cmol}_{\mathrm{c}} \mathrm{dm}^{-3}\right)$ & 6,20 & 82,4 & 85,4 \\
\hline $\mathrm{Na}\left(\mathrm{mg} \mathrm{dm}^{-3}\right)$ & 8,00 & $1.000,0$ & $1.200,0$ \\
\hline $\mathrm{Cu}\left(\mathrm{mg} \mathrm{dm}^{-3}\right)$ & 2,50 & 5,0 & 2,9 \\
\hline $\mathrm{Fe}\left(\mathrm{mg} \mathrm{dm}^{-3}\right)$ & 63,00 & 244,0 & 122,0 \\
\hline $\operatorname{Mn}\left(\mathrm{mg} \mathrm{dm} \mathrm{m}^{-3}\right)$ & 47,00 & 68,0 & 55,0 \\
\hline $\mathrm{Zn}\left(\mathrm{mg} \mathrm{dm}^{-3}\right)$ & 4,40 & 36,0 & 39,0 \\
\hline Matéria orgânica $\left(\mathrm{g} \mathrm{kg}^{-1}\right)$ & 2,30 & 29,9 & 24,2 \\
\hline Condutividade Elétrica $\left(\mu \mathrm{S} \mathrm{cm}^{-3}\right)$ & 184,00 & $1.170,0$ & $1.850,0$ \\
\hline Carbono Orgânico Total $\left(\mathrm{g} \mathrm{kg}^{-1}\right)$ & 1,30 & 17,3 & 14,0 \\
\hline
\end{tabular}

"Vermicomposto (Lc): vermicomposto de lodo de curtume do tipo caleiro.

"Vermicomposto (Lp): vermicomposto de lodo de curtume do tipo primário.

Destaca-se que o lodo de caleiro utilizado no presente estudo refere-se aos resíduos produzidos na etapa de depilação da pele e o lodo primário, àquele proveniente da estação de tratamento primário da indústria curtumeira, os quais formam vermicompostados codispostos com esterco bovino. Na vermicompostagem foram utilizados $80 \%$ de esterco bovino e $20 \%$ do lodo. Para cada tipo de lodo, o processo de vermicompostagem foi realizado separadamente.

Os vermicompostos utilizados neste estudo foram aqueles produzidos a partir da vermicompostagem de substratos constituídos de $20 \%$ de lodo de curtume e $80 \%$ de esterco bovino, os quais foram produzidos conforme descrito em Malafaia et al. (2015b). É importante destacar que a empresa concedente dos lodos trata os efluentes gerados na etapa de curtimento do couro bovino separadamente dos demais resíduos e efluentes produzidos. 
Portanto, os lodos de curtume utilizados neste trabalho não continham metais pesados comumente encontrados em efluentes líquidos das indústrias curtumeiras, como $\mathrm{Cr}, \mathrm{Cd}, \mathrm{Ni}$, $\mathrm{Pb}$ e As. A Tabela 1 apresenta a caracterização desses compostos, realizada segundo as indicações de Tedesco et al. (1995).

O arranjo dos tratamentos consistiu de um fatorial 2x6 (dois tipos de irrigação e seis tipos de adubação), em delineamento inteiramente casualizado (DIC), com cinco repetições, totalizando sessenta unidades experimentais. Foram estabelecidas as seguintes unidades experimentais, irrigadas com água de abastecimento (A) e com água residuária de origem doméstica (R): (T1) Solo controle, sem adubação química e sem vermicomposto; (T2) Solo + NPK; (T3) Solo + vermicomposto de lodo primário; (T4) Solo + P + vermicomposto de lodo primário; (T5) Solo + P + vermicomposto de lodo de caleiro e (T6) Solo + vermicomposto de lodo de caleiro. Destaca-se que o lodo de caleiro utilizado no presente estudo refere-se aos resíduos produzidos na etapa de depilação da pele (que normalmente apresentam sebo, alguns restolhos de carne, resíduos de pele, além de resíduos químicos) e o lodo primário, àquele proveniente do fundo da estação de tratamento primário da indústria curtumeira.

A dose de NPK utilizada nos tratamentos T2(A) e T2(R) foi calculada com base nas necessidades nutricionais da cultura, nas concentrações de nutrientes presentes no solo e na expectativa de rendimento da cultura de $10 \mathrm{Mg} \mathrm{ha}^{-1}$, segundo Sousa e Lobato (2004). As fontes de NPK foram ureia $\left[\left(\mathrm{NH}_{2}\right)_{2} \mathrm{CO}\right]$, superfosfato simples $\left(\mathrm{P}_{2} \mathrm{O}_{5}\right)$ e cloreto de potássio $\left(\mathrm{K}_{2} \mathrm{O}\right)$. Já as quantidades de vermicompostos de lodo de curtume a serem acrescidas ao solo de cultivo foram calculadas com base na concentração do $\mathrm{K}$ (elemento abundante nos vermicompostos utilizados) (Tabela 1), para o fornecimento de $60 \mathrm{~kg} \mathrm{ha}^{-1}$ de $\mathrm{K}_{2} \mathrm{O}$ na base. Assim, a quantidade do vermicomposto de lodo de caleiro acrescida ao solo de cultivo foi equivalente a $6,1 \mathrm{Mg} \mathrm{ha}^{-1}$, e do vermicomposto de lodo primário, 5,5 $\mathrm{Mg} \mathrm{ha}^{-1}$. Não foi necessária a realização da correção do $\mathrm{pH}$ do solo de plantio. A quantidade dos fertilizantes aplicados foi calculada com base no necessário para elevar o teor em cada litro de solo e multiplicado pelo volume de solo do vaso.

O solo, previamente misturado com os vermicompostos (caleiro ou primário) e insumos, foi acondicionado em vasos de polietileno (capacidade volumétrica de $15 \mathrm{~L}$ ) em um total de $12,5 \mathrm{~kg}$. Logo após a instalação das unidades experimentais, os vasos foram semeados com três sementes de milho (Zea mays L.), cultivadas com LG 6036 (LG Semente ${ }^{\circledR}$ ) e, 15 dias depois, realizou-se o desbaste, mantendo-se uma planta por vaso. Os tratos fitossanitários foram realizados quando necessários e a adubação nitrogenada de cobertura (total de $130 \mathrm{~kg}$ $\mathrm{ha}^{-1}$ ) foi realizada superficialmente, em duas parcelas iguais $\left(65 \mathrm{~kg} \mathrm{ha}^{-1}\right)$, aos 40 e 60 dias após a semeadora.

As águas utilizadas nas irrigações foram aquelas provenientes do sistema de abastecimento de água do IF Goiano - Campus Urutaí, tratada em uma Estação de Tratamento de Água (ETA) própria do Campus, e água residuária oriunda de uma lagoa de estabilização de esgoto doméstico, também localizada nas dependências da instituição. Para a caracterização das águas de irrigação, foram coletadas amostras mensais ao longo do período experimental $(n=4)$ para avaliação de parâmetros físicos, químicos e físico-químicos, conforme metodologia proposta pela Apha et al. (1997) (Tabela 2).

O manejo da irrigação da cultura foi realizado a partir de um tanque evaporímetro desenvolvido por Salomão (2012), de forma circular, diâmetro interno de $52 \mathrm{~cm}$ e altura (interna) de $24 \mathrm{~cm}$, instalado sob um estrado de madeira de $15 \mathrm{~cm}$ de altura e instalado no interior do ambiente protegido entre os tratamentos. O volume de água irrigado diariamente, para manter a capacidade de retenção de água do solo em $70 \%\left(243,1 \mathrm{~mL} \mathrm{~kg}^{-1}\right)$ durante o experimento baseou-se na área do vaso a ser irrigado $\left(0,06 \mathrm{~m}^{2}\right)$ e na evapotranspiração da cultura (ETc). Destaca-se que o volume de água a ser reposto era medido em uma proveta graduada. Já a capacidade de retenção de água do solo $\left(\mathrm{C}_{100 \%}=347,4 \mathrm{~mL} \mathrm{~kg}^{-1}\right)$ foi determinada 
por meio do cálculo do poder de embebição do solo, conforme metodologia preconizada pela Embrapa (1997).

Tabela 2. Caracterização física, química e físico-química das águas de irrigação utilizadas no presente estudo, Urutaí, GO, 2016.

\begin{tabular}{|c|c|c|}
\hline Parâmetros & Água de abastecimento* & Água residuária* \\
\hline $\mathrm{pH}$ a $25^{\circ} \mathrm{C}$ & 7,38 & 7,73 \\
\hline Turbidez (UNT) & 2,85 & 601,67 \\
\hline Fe dissolvido $\left(\mathrm{mg} \mathrm{L}^{-1}\right)$ & 0,31 & 1,26 \\
\hline $\mathrm{N}$ total $\left(\mathrm{mg} \mathrm{L}^{-1}\right)$ & 2,43 & 54,57 \\
\hline N orgânico & ND & 4,90 \\
\hline $\mathrm{N}$ amonical $\left(\mathrm{mg} \mathrm{L}^{-1}\right)$ & 0,07 & 43,67 \\
\hline Nitrato $\left(\mathrm{mg} \mathrm{L}^{-1}\right)$ & 0,33 & 6,00 \\
\hline Condutividade elétrica a $25^{\circ} \mathrm{C}\left(\mu \mathrm{s} \mathrm{cm}^{-1}\right)$ & 67,20 & 746,33 \\
\hline$P$ total $\left(\mathrm{mg} \mathrm{L}^{-1}\right)$ & 0,14 & 9,10 \\
\hline Ortofosfato $\left(\mathrm{mg} \mathrm{L}^{-1}\right)$ & 0,33 & 20,86 \\
\hline $\mathrm{DBO}\left(\mathrm{mg} \mathrm{L}^{-1}\right)$ & 0,67 & 572,11 \\
\hline Sólidos totais $\left(\mathrm{mg} \mathrm{L}^{-1}\right)$ & 73,33 & $1.290,00$ \\
\hline $\mathrm{Cu}$ dissolvido $\left(\mathrm{mg} \mathrm{L}^{-1}\right)$ & 0,35 & 0,44 \\
\hline $\mathrm{Zn}\left(\mathrm{mg} \mathrm{L}^{-1}\right)$ & 0,37 & 0,26 \\
\hline $\mathrm{Na}\left(\mathrm{mg} \mathrm{L}^{-1}\right)$ & 25,59 & 56,63 \\
\hline Mn dissolvido $\left(\mathrm{mg} \mathrm{L}^{-1}\right)$ & 0,57 & 1,80 \\
\hline $\mathrm{Mg}$ dissolvido $\left(\mathrm{mg} \mathrm{L}^{-1}\right)$ & 1,78 & 12,16 \\
\hline $\mathrm{Ca}\left(\mathrm{mg} \mathrm{L}^{-1}\right)$ & 6,28 & 37,41 \\
\hline $\mathrm{S}\left(\mathrm{mg} \mathrm{L}^{-1}\right)$ & 3,00 & 3,10 \\
\hline $\mathrm{K}\left(\mathrm{mg} \mathrm{L}^{-1}\right)$ & 7,45 & 19,16 \\
\hline $\operatorname{COT}\left(\mathrm{mg} \mathrm{L}^{-1}\right)$ & 14,95 & 43,47 \\
\hline
\end{tabular}

Legenda: ND: parâmetro não dosado.

"Os valores apresentados referem-se à média de quatro amostras coletadas ao longo do período experimental.

Para a análise do tecido foliar, coletou-se, de cada planta, a folha inteira oposta e abaixo da primeira espiga (superior), excluída a nervura central, por ocasião do aparecimento da inflorescência feminina (Malavolta et al., 1997). As folhas foram lavadas em água destilada (corrente), acondicionadas em sacos de papel e secas em temperatura de $65^{\circ} \mathrm{C}$ até que a massa ficasse constante para determinação dos teores de $\mathrm{N}, \mathrm{P}, \mathrm{K}, \mathrm{Ca}, \mathrm{Mg}, \mathrm{Cu}, \mathrm{Fe}, \mathrm{Mn}$ e $\mathrm{Zn}$, conforme metodologia proposta pela Embrapa (2009).

Todos os dados foram submetidos à análise de variância de acordo com o modelo fatorial (two-way ANOVA), sendo os fatores tratamentos (seis níveis) e irrigação (dois níveis), com cinco repetições. Nos casos de F significativo, foi aplicado o teste de Tukey a 5\% de probabilidade. Destaca-se que a análise de variância foi realizada por meio do software ASSISTAT, versão 7.7 beta (cópia distribuída gratuitamente). 


\section{RESULTADOS E DISCUSSÃO}

Sabe-se que a análise foliar constitui importante prática para o monitoramento do estado nutricional das plantas. No presente estudo, observou-se que houve interação entre as fontes de variação "irrigação" e "tipos de adubação" para as concentrações de todos os macro e micronutrientes avaliados nas folhas das plantas de milho (Tabela 3 ).

Tabela 3. Resumo do teste $\mathrm{F}$ da análise de variância para as variáveis macro e micronutrientes nas folhas do milho (Zea mays L. - LG 6036), em função do tipo de água de irrigação e dos tratamentos de adubação, Urutaí, GO, 2014.

\begin{tabular}{|c|c|c|c|c|}
\hline Causa da variação & $\begin{array}{c}\text { Fator } 1 \\
\text { (tipos de irrigação) }\end{array}$ & $\begin{array}{c}\text { Fator } 2 \\
\text { (tratamentos) }\end{array}$ & $\begin{array}{c}\text { Interação } \\
\text { (fator } 1 \times \text { fator 2) }\end{array}$ & $\begin{array}{l}\mathrm{CV} \\
(\%)\end{array}$ \\
\hline \multirow{5}{*}{$\mathrm{g} \mathrm{kg}^{-1}$} & $250,24 * *$ & $438,33 * *$ & $42,46 * *$ & 3,76 \\
\hline & $2.368,81 * *$ & $1.652,69 * *$ & $48,05 * *$ & 3,22 \\
\hline & $514,94 * *$ & $384,21 * *$ & $65,84 * *$ & 1,33 \\
\hline & $366,67 * *$ & $93,92 * *$ & $73,25 * *$ & 2,55 \\
\hline & $641,32 * *$ & $164,54 * *$ & $170,22 * *$ & 2,62 \\
\hline \multirow{4}{*}{$\mathrm{mg} \mathrm{kg}^{-1}$} & $103,91 * *$ & $176,98 * *$ & $121,82 * *$ & 4,98 \\
\hline & $1.9758,91 * *$ & $4.2651,16^{* *}$ & $1.129,52 * *$ & 0,37 \\
\hline & $784,72 * *$ & $2.050,90^{* *}$ & $139,90 * *$ & 2,47 \\
\hline & $2.112,47 * *$ & $815,76^{* *}$ & $269,05 * *$ & 2,40 \\
\hline
\end{tabular}

Legenda: ${ }^{* *}$ significativo a $1 \%$ de probabilidade;

CV é o coeficiente de variação, expresso em porcentagem.

Tipos de irrigação: água de abastecimento e água residuária de origem doméstica.

Alguns elementos como $\mathrm{Ca}, \mathrm{Fe}$ e o $\mathrm{P}$, principalmente nos grupos irrigados com água residuária, apresentaram concentrações acima da faixa de referência, o que pode ser considerado como um consumo de luxo pela planta, uma vez que as mesmas não apresentaram nenhum sintoma de toxidez aparente. No entanto, para a maioria dos macro e micronutrientes nos demais grupos avaliados, os valores se encontraram dentro da faixa de referência considerada adequada para nutrição da cultura do milho (Büll, 1993) (Tabela 4).

Para todos os nutrientes avaliados, incluindo aqueles mais exigidos pela cultura do milho, como o $\mathrm{N}$ e o $\mathrm{P}$ (Coelho, 2008), a análise foliar revelou que os tratamentos irrigados com água residuária apresentaram maiores valores em relação aos tratamentos irrigados com água de abastecimento (Tabela 5). Esses resultados podem ser explicados pela maior biodisponibilidade dos elementos na água residuária, conforme demonstrado também em outros estudos (Almeida et al., 2012; Costa et al., 2012; Andrade-Filho et al., 2013; Rocha et al. 2014).

Na Tabela 2 pode-se observar que a água residuária continha valores mais elevados de todos os elementos (exceto o $\mathrm{Zn}$ ) quando comparada à água de abastecimento. Essas elevadas concentrações podem ter aumentado a disponibilidade dos elementos no solo e, consequentemente, facilitado a sua assimilação pelas plantas. Outro fator, que pode ter influenciado na boa disponibilidade de alguns elementos para as plantas, é a baixa concentração de matéria orgânica no solo de cultivo, caso dos elementos como $\mathrm{Cu}, \mathrm{Fe}$ e $\mathrm{Mn}$. 
Tabela 4. Resultado da análise foliar de milho (Zea mays L.) cultivado em tratamentos, contendo vermicomposto de lodo de curtume e irrigação com água residuária de esgoto doméstico e sua comparação com os limites estabelecidos como adequados para a cultura. Urutaí, GO, 2014.

\begin{tabular}{|c|c|c|c|c|c|c|c|c|c|}
\hline & $\mathbf{N}$ & $\mathbf{P}$ & $\mathbf{K}$ & $\mathbf{C a}$ & Mg & $\mathbf{C u}$ & $\mathbf{F e}$ & Mn & $\mathbf{Z n}$ \\
\hline & \multicolumn{5}{|c|}{ - } & \multicolumn{4}{|c|}{------ $\mathrm{mg} \mathrm{kg}^{-1}$} \\
\hline Faixa de referência $^{1}$ & $\begin{array}{c}2,75- \\
3,25\end{array}$ & $\begin{array}{c}0,19- \\
0,35\end{array}$ & $\begin{array}{l}1,75- \\
2,97\end{array}$ & $\begin{array}{c}0,23- \\
0,40\end{array}$ & $\begin{array}{c}0,15- \\
0,40\end{array}$ & $\begin{array}{l}6- \\
20\end{array}$ & $\begin{array}{l}50- \\
250\end{array}$ & $\begin{array}{l}42- \\
150\end{array}$ & $15-50$ \\
\hline \multicolumn{10}{|c|}{ Tratamentos irrigados com água de abastecimento } \\
\hline Solo - testemunha & $\boldsymbol{\nabla}$ & $\boldsymbol{\nabla}$ & $\bullet$ & $\boldsymbol{\Delta}$ & $\bullet$ & $\bullet$ & $\bullet$ & $\bullet$ & $\bullet$ \\
\hline Solo + NPK & $\bullet$ & $\boldsymbol{\nabla}$ & $\bullet$ & $\bullet$ & $\bullet$ & $\bullet$ & $\bullet$ & $\bullet$ & $\bullet$ \\
\hline Solo + Lp & $\boldsymbol{\nabla}$ & $\bullet$ & $\bullet$ & $\Delta$ & $\bullet$ & $\bullet$ & $\boldsymbol{\Delta}$ & $\bullet$ & $\bullet$ \\
\hline Solo + P + Lp & $\bullet$ & $\bullet$ & $\bullet$ & $\boldsymbol{\Delta}$ & $\bullet$ & $\bullet$ & $\boldsymbol{\Delta}$ & $\bullet$ & $\bullet$ \\
\hline Solo + Lc & $\bullet$ & $\bullet$ & $\bullet$ & $\boldsymbol{\Delta}$ & $\bullet$ & $\bullet$ & $\boldsymbol{\Delta}$ & $\bullet$ & $\bullet$ \\
\hline Solo + P + Lc & $\bullet$ & $\boldsymbol{\Delta}$ & $\bullet$ & $\boldsymbol{\Delta}$ & $\bullet$ & $\bullet$ & $\Delta$ & $\bullet$ & $\bullet$ \\
\hline
\end{tabular}

Tratamentos irrigados com água residuária

\begin{tabular}{|c|c|c|c|c|c|c|c|c|}
\hline Solo - testemunha & $\bar{\nabla}$ & $\boldsymbol{\nabla}$ & $\bullet$ & $\Delta$ & $\bullet$ & • & $\bullet$ & $\bar{\nabla}$ \\
\hline Solo + NPK & $\bullet$ & $\bullet$ & $\bullet$ & $\boldsymbol{\Delta}$ & $\bullet$ & $\bullet$ & $\bullet$ & $\bullet$ \\
\hline Solo + Lp & $\bullet$ & $\Delta$ & $\bullet$ & $\boldsymbol{\Delta}$ & $\bullet$ & $\Delta$ & $\boldsymbol{\Delta}$ & $\bullet$ \\
\hline Solo + P + Lp & $\bullet$ & $\Delta$ & $\bullet$ & $\boldsymbol{\Delta}$ & $\bullet$ & $\bullet$ & $\boldsymbol{\Delta}$ & $\bullet$ \\
\hline Solo + Lc & $\bullet$ & $\Delta$ & $\bullet$ & $\boldsymbol{\Delta}$ & $\bullet$ & $\bullet$ & $\boldsymbol{\Delta}$ & $\bullet$ \\
\hline Solo $+\mathrm{P}+\mathrm{Lc}$ & $\boldsymbol{\Delta}$ & $\boldsymbol{\Delta}$ & $\bullet$ & $\boldsymbol{\Delta}$ & $\bullet$ & $\bullet$ & $\boldsymbol{\Delta}$ & $\bullet$ \\
\hline
\end{tabular}

${ }^{1}$ Os teores foliares de macro e micronutrientes considerados faixa de referência, foram compilados de diversos autores por Büll (1993).

Legenda: • - concentração dentro do intervalo: nutrição considerada adequada;

$\Delta$ - concentração acima do limite superior: absorção de luxo ou, se apresentar sintomas, toxidez;

$\boldsymbol{\nabla}$ - concentração abaixo do limite inferior: deficiência do nutriente.

Lp _ vermicomposto de lodo de curtume do tipo primário;

Le _ vermicomposto de lodo de curtume do tipo caleiro.

Em relação aos tipos de adubação, observou-se que todos os tratamentos, irrigados ou não com água residuária, apresentaram concentrações foliares maiores do que todos os nutrientes avaliados, que as observadas nas folhas do tratamento T1 (controle). Além disso, cabe salientar que as plantas dos tratamentos T3R, T4R, T5R e T6R (nos quais os solos foram acrescidos com vermicompostos de lodo de curtume), irrigadas com água residuária, apresentaram concentrações de alguns nutrientes como $\mathrm{N}, \mathrm{K}, \mathrm{Ca}$ e $\mathrm{Zn}$, similares às das plantas do tratamento T2R, o qual recebeu adubação química (Tabela 5), ou maiores, caso do $\mathrm{P}, \mathrm{Mg}$, $\mathrm{Cu}, \mathrm{Fe}$ e $\mathrm{Mn}$. Esses resultados podem ser explicados pela maior concentração do $\mathrm{N}$ presente nos vermicompostos de lodo de curtume utilizados, quando comparada à concentração inicial do solo de cultivo (Tabela 1).

Os resultados encontrados corroboram estudos anteriores que demonstraram que a irrigação da cultura do milho com água residuária (Al-Jaloud et al., 1995) ou o cultivo da planta em solo acrescido com vermicompostos (Theunissen et al., 2010; Kalantari et al., 2010), proporcionam aumento da concentração foliar de vários macro e micronutrientes. De todo modo, é importante salientar que o presente estudo é pioneiro em avaliar teores de macro e micronutrientes foliares em plantas de milho cultivadas em solo fertilizado com vermicompostos de lodo de curtume e irrigado com água residuárias (em associação), fato 
este nunca demonstrado na literatura.

Tabela 5. Valores médios do desdobramento da interação tipo de água de irrigação $x$ tratamentos de adubação para os teores de macro e micronutrientes nas folhas do milho (LG 6036). Urutaí, GO, 2014.

\begin{tabular}{|c|c|c|c|c|c|c|}
\hline \multirow[b]{2}{*}{$\begin{array}{l}\text { Tipos de água } \\
\text { irrigação }\end{array}$} & \multicolumn{6}{|c|}{ Tratamentos } \\
\hline & $\begin{array}{c}\text { Solo } \\
\text { (testemunha) } \\
\text { (T1) }\end{array}$ & $\begin{array}{c}\text { Solo + } \\
\text { NPK (T2) }\end{array}$ & $\begin{array}{l}\text { Solo + Lp } \\
\quad \text { (T3) }\end{array}$ & $\begin{array}{c}\text { Solo + P } \\
+ \text { Lp (T4) }\end{array}$ & $\begin{array}{l}\text { Solo + Lc } \\
\text { (T5) }\end{array}$ & $\begin{array}{l}\text { Solo + P } \\
+ \text { Lc (T6) }\end{array}$ \\
\hline \multicolumn{7}{|c|}{$\mathrm{N}\left(\mathrm{g} \mathrm{kg}^{-1}\right)$} \\
\hline Abastecimento & $0,80 \mathrm{bC}$ & $3,00 \mathrm{bA}$ & $2,50 \mathrm{bB}$ & $3,06 \mathrm{bA}$ & $3,12 \mathrm{aA}$ & $3,04 \mathrm{bA}$ \\
\hline Residuária & $2,00 \mathrm{aB}$ & $3,18 \mathrm{aA}$ & $3,20 \mathrm{aA}$ & $3,22 \mathrm{aA}$ & $3,24 \mathrm{aA}$ & $3,26 \mathrm{aA}$ \\
\hline \multicolumn{7}{|c|}{$\mathrm{P}\left(\mathrm{g} \mathrm{kg}^{-1}\right)$} \\
\hline Abastecimento & $0,08 \mathrm{bF}$ & $0,17 \mathrm{bE}$ & $0,24 \mathrm{bD}$ & $0,28 \mathrm{bC}$ & $0,33 \mathrm{bB}$ & $0,37 \mathrm{bA}$ \\
\hline Residuária & $0,13 \mathrm{aF}$ & $0,27 \mathrm{aE}$ & $0,37 \mathrm{aD}$ & $0,43 \mathrm{aC}$ & $0,49 \mathrm{aB}$ & $0,53 \mathrm{aA}$ \\
\hline \multicolumn{7}{|c|}{$\mathrm{K}\left(\mathrm{g} \mathrm{kg}^{-1}\right)$} \\
\hline Abastecimento & $2,06 \mathrm{bE}$ & $2,24 \mathrm{bD}$ & $2,56 \mathrm{bB}$ & $2,48 \mathrm{bC}$ & $2,75 \mathrm{bA}$ & $2,56 \mathrm{aB}$ \\
\hline Residuária & $2,40 \mathrm{aD}$ & $2,56 \mathrm{aC}$ & $2,96 \mathrm{aA}$ & $002,56 \mathrm{aC}$ & $2,80 \mathrm{aB}$ & $2,56 \mathrm{aC}$ \\
\hline \multicolumn{7}{|c|}{$\mathrm{Ca}\left(\mathrm{g} \mathrm{kg}^{-1}\right)$} \\
\hline Abastecimento & $0,44 b C$ & $0,39 \mathrm{bD}$ & $0,47 b C$ & $0,51 \mathrm{bB}$ & $0,58 \mathrm{aA}$ & $0,56 \mathrm{bA}$ \\
\hline Residuária & $0,55 \mathrm{aCD}$ & $0,53 \mathrm{aDE}$ & $0,57 \mathrm{aBC}$ & $0,61 \mathrm{aA}$ & $0,52 \mathrm{bE}$ & $0,58 \mathrm{aB}$ \\
\hline \multicolumn{7}{|c|}{$\operatorname{Mg}\left(\mathrm{g} \mathrm{kg}^{-1}\right)$} \\
\hline Abastecimento & $0,21 \mathrm{bC}$ & $0,22 \mathrm{bC}$ & $0,19 \mathrm{bD}$ & $0,28 \mathrm{bB}$ & $000,33 \mathrm{aA}$ & $000,27 \mathrm{bB}$ \\
\hline Residuária & $0,28 \mathrm{aD}$ & $0,26 \mathrm{aE}$ & $0,34 \mathrm{aA}$ & $0,32 \mathrm{aB}$ & $000,30 \mathrm{bC}$ & $000,29 \mathrm{aCD}$ \\
\hline \multicolumn{7}{|c|}{$\mathrm{Cu}\left(\mathrm{mg} \mathrm{kg}^{-1}\right)$} \\
\hline Abastecimento & $11,20 \mathrm{bD}$ & $11,00 \mathrm{bD}$ & $13,00 \mathrm{bC}$ & $019,00 \mathrm{aA}$ & $016,00 \mathrm{aB}$ & $014,00 \mathrm{bC}$ \\
\hline Residuária & $13,00 \mathrm{aD}$ & $12,00 \mathrm{aD}$ & $25,00 \mathrm{aA}$ & $018,00 \mathrm{bB}$ & $013,00 \mathrm{bD}$ & $015,00 \mathrm{aC}$ \\
\hline \multicolumn{7}{|c|}{$\mathrm{Fe}\left(\mathrm{mg} \mathrm{kg}^{-1}\right)$} \\
\hline Abastecimento & 190,00bEX & $190,00 \mathrm{bE}$ & $280,00 \mathrm{bD}$ & $360,00 \mathrm{bA}$ & $330,00 \mathrm{bC}$ & $340,00 \mathrm{bB}$ \\
\hline Residuária & $240,00 \mathrm{aFX}$ & $250,00 \mathrm{aE}$ & $290,00 \mathrm{aD}$ & $370,00 \mathrm{aC}$ & $380,00 \mathrm{aB}$ & $399,60 \mathrm{aA}$ \\
\hline \multicolumn{7}{|c|}{$\operatorname{Mn}\left(\mathrm{mg} \mathrm{kg}^{-1}\right)$} \\
\hline Abastecimento & $45,80 \mathrm{aDX}$ & $45,60 \mathrm{bD}$ & $84,00 \mathrm{bB}$ & $079,80 \mathrm{bC}$ & $111,60 \mathrm{bA}$ & $113,00 \mathrm{bA}$ \\
\hline Residuária & $34,00 \mathrm{bDX}$ & $83,00 \mathrm{aC}$ & $103,40 \mathrm{aB}$ & $103,00 \mathrm{aB}$ & $124,60 \mathrm{aA}$ & $125,80 \mathrm{aA}$ \\
\hline \multicolumn{7}{|c|}{$\mathrm{Zn}\left(\mathrm{mg} \mathrm{kg}^{-1}\right)$} \\
\hline Abastecimento & $18,40 \mathrm{bEX}$ & $20,00 \mathrm{bD}$ & $21,00 \mathrm{bD}$ & $042,00 \mathrm{aA}$ & $033,00 \mathrm{aB}$ & $027,00 \mathrm{bC}$ \\
\hline Residuária & $26,00 \mathrm{aDX}$ & $33,00 \mathrm{aC}$ & $40,00 \mathrm{aB}$ & $042,00 \mathrm{aA}$ & $033,00 \mathrm{aC}$ & $041,00 \mathrm{aAB}$ \\
\hline
\end{tabular}

Em relação ao Fe em especial, notou-se que as folhas das plantas dos tratamentos T3, T4, T5 e T6, irrigadas ou não com água residuária, apresentaram valores elevados e superiores ao limite máximo considerado adequado para a cultura, sem apresentar sintomas de toxidez (Tabela 4). As concentrações de $\mathrm{Fe}$ encontrados nos referidos grupos foram superiores estatisticamente às encontradas nos tratamentos $\mathrm{T} 1$ (testemunha) e T2 (o qual recebeu adubação química) (Tabela 5).

Esses resultados devem-se, possivelmente, às concentrações de $\mathrm{Fe}$ elevadas nos vermicompostos utilizados em relação à concentração inicial do elemento no solo de cultivo (Tabela 1). É importante salientar que, embora estudos tenham demonstrando que a absorção 
do Fe pelas plantas pode ser influenciada por outros nutrientes, como o $\mathrm{K}, \mathrm{Ca}$ e $\mathrm{Mg}$ (Malavolta et al. 1997; Borges et al., 2007), no presente estudo não foi evidenciada baixa disponibilidade do $\mathrm{Fe}$ nas plantas em consequência do acréscimo de outros nutrientes via adição de vermicompostos no solo de cultivo e, ou, irrigação com água residuária.

\section{CONCLUSÃO}

Conclui-se com este estudo que tanto a água residuária doméstica, quanto os vermicompostos de lodo de curtume utilizados constituem boa fonte de nutrientes para as plantas de milho, uma vez que os teores de macro e micronutrientes identificados nas folhas mostraram-se satisfatórios, não tendo sido observado nenhum sinal ou sintoma de toxidez. Embora a análise foliar não deva servir como ferramenta exclusiva para avaliação do estado nutricional das plantas, este estudo, pioneiro em observar a existência de interação entre os fatores estudados, aponta para a potencialidade da utilização dos resíduos escolhidos na cultura do milho, motivando a realização de novas pesquisas.

\section{REFERÊNCIAS}

AL-JALOUD, A. A.; HUSSAIN, G.; AL-SAATI, A. J.; KARIMULLA, S. Effect of wastewater irrigation on mineral composition of corn and sorghum plants in a pot experiment. Journal of Plant Nutrition, v. 18, n. 8, p. 1677-1692, 1995. http://dx.doi.org/10.1080/01904169509365013

ALMEIDA, J. P. N.; COSTA, L. R.; SAMPAIO, P. R. F.; AZEVEDO, J.; DIAS, N. S. Utilização de esgoto doméstico tratado na produção de mudas de maracujazeiro amarelo. Revista Verde de Agroecologia e Desenvolvimento Sustentável, v. 7, n. 4, p. 69-75, 2012.

ANDRADE-FILHO, J. A.; SOUSA NETO, O. N.; DIAS, N. S.; NASCIMENTYO, I. B.; MEDEIROS, J. F.; COSME, C. R. Atributos químicos de solo fertirrigado com água residuária no semiárido brasileiro. Irriga, v. 18, n. 4, p. 661-674, 2013. http://dx.doi.org/10.15809/irriga.2013v18n4p661

AMERICAN PUBLIC HEALTH ASSOCIATION - APHA; AMERICAN WATER WORKS ASSOCIATION - AWWA; WATER ENVIRONMENT FEDERATION - WEF. Standard methods for the examination of water and wastewater. 20. ed. New York, 1997. $1194 \mathrm{p}$.

BONINI, M. A.; SATO, L. M.; BASTOS, R. G.; SOUZA, C. F. Alterações nos atributos químicos e físicos de um Latossolo Vermelho irrigado com água residuária e vinhaça. Revista Biociências, v. 20, n. 1, p. 56-63, 2014.

BORGES, J. D.; BARROS, R. G.; SOUZA, E. R. B.; OLIVEIRA-JÚNIOR, J. P.; LEANDRO, W. M.; OLIVEIRA, I. P. et al. Teores de micronutrientes nas folhas de milho fertilizadas com lodo de curtume. Bioscience Journal, v. 23, n. 2, p. 1-6, 2007.

BÜLL, L. T. Nutrição mineral do milho. In: BÜLL, L. T.; CANTARELA, H. (Ed.). Cultura do milho: fatores que afetam a produtividade. Piracicaba: Associação Brasileira para Pesquisa da Potassa e do Fostato, 1993. p. 63-145.

COELHO, A. M. Nutrição e Adubação do Milho. In: CRUZ, J. C.; KARAM, D.; MONTEIRO, M. A. R.; MAGALHÃES, P. C. (Org.). A cultura do milho. Sete Lagoas: Embrapa Milho e Sorgo, 2008. Vol. 1. p. 131-157. 
COSTA, M. S.; COSTA, Z. V. B.; ALVES, S. M. C.; NETO, M. F.; MARINHO, M. J. C. Avaliação nutricional do milho cultivado com diferentes doses de efluente doméstico tratado. Irriga, v. 1, n. 1, p. 12-26, 2012. http://dx.doi.org/10.15809/irriga. 2012v1n01p12

EMPRESA BRASILEIRA DE PESQUISA AGROPECUÁRIA - EMBRAPA. Sistema brasileiro de classificação de solos. 3. ed. Brasília, 2013. 353p.

EMPRESA BRASILEIRA DE PESQUISA AGROPECUÁRIA - EMBRAPA. Centro Nacional de Pesquisa de Solos. Manual de métodos de análise de solo. 2. ed. Brasília, 1997. $212 \mathrm{p}$.

EMPRESA BRASILEIRA DE PESQUISA AGROPECUÁRIA - EMBRAPA. Manual de análises químicas de solos, plantas e fertilizantes. 2. ed. Brasília, 2009. 627 p.

FONSECA, A. F.; LEAL, R. M. P.; HERPIN, U.; MELFI, A. J. Carbon and nitrogen dynamics in a Brazilian soil-pasture system irrigated with treated sewage effluent. Israel Journal of Plant Sciences, v. 59, n. 2-4, p. 147-157, 2011. http://dx.doi.org/ 10.1560/IJPS.59.2-4.147

GÖDECKE, M. V.; RODRIGUES, M. A. S.; NAIME, R. H. Resíduos de curtume: estudo das tendências de pesquisa. Revista Eletrônica em Gestão, Educação e Tecnologia Ambiental, v. 7, n. 7, p. 1357-1378, 2012. http://dx.doi.org/10.5902/223611705779

KALANTARI, S.; HATAMI, S.; ARDALAN, M. M.; ALIKHANI, H. A.; SHARAFA, M. The effect of compost and vermicompost of yard leaf manure on growth of corn. African Journal of Agricultural Research, v. 5, n. 11, p. 1317-1323, 2010.

KRAEMER, M. E. P. A questão ambiental e os resíduos industriais. Disponível em: http://www.amda.org.br/imgs/up/Artigo_25.pdf. Acesso em: 14 nov. 2014.

LEAL, R. M. P.; FONSECA, A. F.; HERPIN, U.; MELFI, A. J. Agricultural utilization of treated sewage effluent: experience from Brazil. Israel Journal of Plant Science, v. 59, n. 2-4, p. 235-248, 2011. http://dx.doi.org/10.1560/IJPS.59.2.xx

LUERSEN, L. H.; SHULTZ, G.; REMPEL, C. Reuso do efluente tratado na indústria curtidora. Tecno-Lógica, v. 16, n. 1, p. 5-10, 2012. http://dx.doi.org/10.17058/ tecnolog.v16i1.2311

MALAFAIA, G.; JORDÃO, C. R.; ARAÚJO, F. G.; LEANDRO, W. M.; RODRIGUES, A. S. L. Vermicompostagem de lodo de curtume em associação com esterco bovino utilizando Eisenia foetida. Engenharia Sanitária e Ambiental, v. 20, n. 4, p. 709-716, 2015a. http://dx.doi.org/10.1590/S1413-41522015020040134645

MALAFAiA, G.; ESTRELA, D. C.; GUIMARÃES, A. B. T.; ARAÚJO, F. G.; LEANDRO, W. M.; RODRIGUES, A. S. L. Vermicomposting of diferente types of tanning sludge (liming and primary) mixed with cattel dung. Ecological Engineering, v. 85, p. 301306, 2015b. http://dx.doi.org/10.1016/j.ecoleng.2015.10.010

MALAVOLTA, E.; VITTI, G. C.; OLIVEIRA, S. A. Avaliação do estado nutricional das plantas: princípios e aplicações. Piracicaba: POTAFOS, 1997. 201p. 
MONTEIRO, D. R.; SILVA, T. T. S.; SILVA, L. V. B. D.; LIMA, V. L. A.; SANTOS, C. L. M.; PEARSON, H. W. efeito da aplicação de efluente doméstico tratado nos teores de micronutrientes no solo. Irriga, v. Especial, n. Especial, p. 40-46, 2014. http://dx.doi.org/10.15809/irriga.2014v1n1p40

NUNES, P. M. P.; SMOLAREK, F. S. F.; KAMINSKI, G. A. T.; FIN, M. T.; ZANIN, S. M. W.; MIGUEL, M. D. et al. A importância do aproveitamento dos resíduos industriais da semente de Citrus. Visão Acadêmica, v. 10, n. 1, p. 97-110, 2009. http://dx.doi.org/10.5380/acd.v10i1.21324

ROCHA, S. A.; GARCIA, G. O.; LOUGON, M. S.; CECÍLIO, R. A.; CALDEIRA, M. V. W. Growth and nutrition leaf of Eucalyptus sp. due to use the different water qualities. Revista de Ciências Agrárias, v. 37, n. 2, p. 141-151, 2014.

SALOMÃO, L. C. Calibração de tanques evaporímetros de baixo custo sob diferentes diâmetros em ambiente protegido. 2012. 74f. Tese (Doutorado em Agronomia) Faculdade de Ciências Agronômicas da Universidade Estadual Paulista Júlio de Mesquita Filho, Botucatu, 2012.

SILVA, L. L.; CARVALHO, C. M.; SOUZA, R. P. F.; FEITOSA, H. O.; FEITOSA, S. O.; FILHO, R. R. G. Utilização de efluentes domésticos no crescimento da pimenta (Capsicum chinense), cultivar tequila bode vermelha. Revista AGROTEC, v. 35, n. 1, p. 121-133, 2014.

SOUSA, D. M. G.; LOBATO, E. Calagem e adubação para culturas anuais e semiperenes. In: SOUSA, D. M. G.; LOBATO E. (Ed.). Cerrado: correção do solo e adubação. 2. ed. Planaltina: Embrapa Cerrado, 2004. p. 283-315.

TEDESCO, M. J.; GIANELlO, C.; BISSANI, C.; BOHNEN, H.; VOLKWEISS, S. J. Análise de solo, plantas e outros materiais. 2. ed. Porto Alegre: Universidade Federal do Rio Grande do Sul, 1995. 174 p.

THEUNISSEN, J.; NDAKIDEMI, P. A.; LAUBSCHER, C. P. Potential of vermicompost produced from plant waste on the growth and nutrient status in vegetable production. International Journal of the Physical Sciences, v. 5, n. 13, p. 1964-1973, 2010.

VIG, A. P.; SINGH, J.; WANI, S. H.; DHALIWAL, S. S. Vermicomposting of tannery sludge mixed with cattle dung into valuable manure using earthworm Eisenia fetida (Savigny). Bioresource Technology, v. 102, n. 17, p. 7941-7945, 2011. http://dx.doi.org/10. 1016/j.biortech.2011.05.056 